\title{
Automatic Vessel Lumen Segmentation in Optical Coherence Tomography (OCT) Images
}

\author{
Huaizhong Zhang ${ }^{a}$, Ehab Essa ${ }^{b}$, Xianghua Xie ${ }^{b}$ \\ ${ }^{a}$ Department of Computer Science, Edgehill University, Ormskirk, UK, L39 4QP \\ ${ }^{b}$ Department of Computer Science, Swansea University, UK, SA2 8PP
}

\begin{abstract}
This paper presents a graph based method to automatically and accurately segment the lumen borders from optical coherence tomography (OCT) images. The proposed method unravels the OCT images from the Cartesian coordinates to polar coordinates so that the segmentation is transferred into a height field delineation problem. In effect, the method imposes a simplistic star shape prior but without the bias towards narrower lumen size. The lumen border is identified as the solution to finding the minimum closed set on a node weighed, directed graph. In order to cope with both the variability in imaging condition and different forms of image artefacts, we adopt an image feature that relies on very little assumption on the appearance of lumen border but is resilient to image noise and so on. This feature is derived from a convolution of the image gradient field and thus it takes into account gradient vector interactions at a much more global scale compared to conventional gradient based approaches. The proposed method is fully automatic without the need for an initialisation. We compare this method with a number of techniques, including both conventional methods and data driven models.
\end{abstract} Keywords: image segmentation; gradient convolution field; star prior; 
optical coherence tomography; graph cuts

\section{Introduction}

Optical coherence tomography (OCT) has become a prominent intravascilar imaging technique since its introduction several decades ago [1] and has been routinely used in clinics, particularly in tissue imaging requiring high resolution such as in coronary disease diagnosis and treatment [2]. OCT imaging has been used as a reference tool for patient follow-up, identification of vulnerable plaque, quantification of in-stent neointimal hyperplasia and guiding optimal antiplatelet therapy to prevent late stent thrombosis $[3,4]$. A typical OCT image usually consists of three parts as shown in Fig. 1(a), which are lumen, vessel with intima and media layers, and adventitia surrounding the vessel wall. Among these regions of interest (ROIs), the lumen area is one of the major concerns in OCT in order to provide essential information for reconstructing the coronary arteries to help diagnose cardiovascular diseases such as atherosclerosis $[2,5]$.

Due to factors such as high signal attenuation, catheter and stent interference, and bifurcation, OCT imaging however suffers from various forms of artefacts. Fig. 1(a-c) illustrate three examples of these difficulties. The lumen delineation in OCT can provide accurate morphological information of the luminal cross-sectional area and enable its quantification and visualization, which is crucial in conducting plaque segmentation and assessing stent apposition [6, 7]. Manual delineation however is a time-consuming, cumbersome procedure and lacks consistency due to subjectivity. Motivated by the fact that these limitations substantially reduce the efficiency and effective- 
ness of lumen identification, semi-automated approaches or even automatic ones are of particular interest in lumen segmentation.

In the past decade, a variety of methods have been proposed to carry out lumen segmentation in OCT. However, many of those methods suffer from local minima and severe artefacts in OCT, which can lead to unsatisfactory segmentation results, for examples, over-smoothing as shown in spline-fitting [8] and Markov-random field models [5], contour discontinuity and time consuming for the light back-scattering method [9] (18.82 seconds per frame $(\mathrm{s} / \mathrm{f}))$ and mathematical morphology [10] (5.9 s/f). Graph cut based methods are atractive alternatives because of their global solution schemes and simple user interaction $[4,11,12,13]$. Among many others, the $s-t$ cut [14] is a commonly used method by calculating a cut from source subset and sink subset in a derived graph, which can be implemented with the mincut/max-flow algorithms $[15,16,17]$. Typically, the functional energy of the $s-t$ cut is formulated by combining the user interaction term (data) and the regularisation term (prior), and then the augmenting-path algorithm [16] is employed to numerically implement the generated formulation. In addition, due to image data features generated with user interaction, the obtained object representation can lead to more accurate segmentation. However, there are a few challenges in applying this method to OCT segmentation. First, the performance of the $s-t$ cut is subject to the accuracy of prior information; second, the obtained shape are sensitive to initial seed selections; third, most importantly, intensive user interactions may significantly reduce segmentation efficiency.

Shape prior has been proposed in graph cut methods in order to minimise 


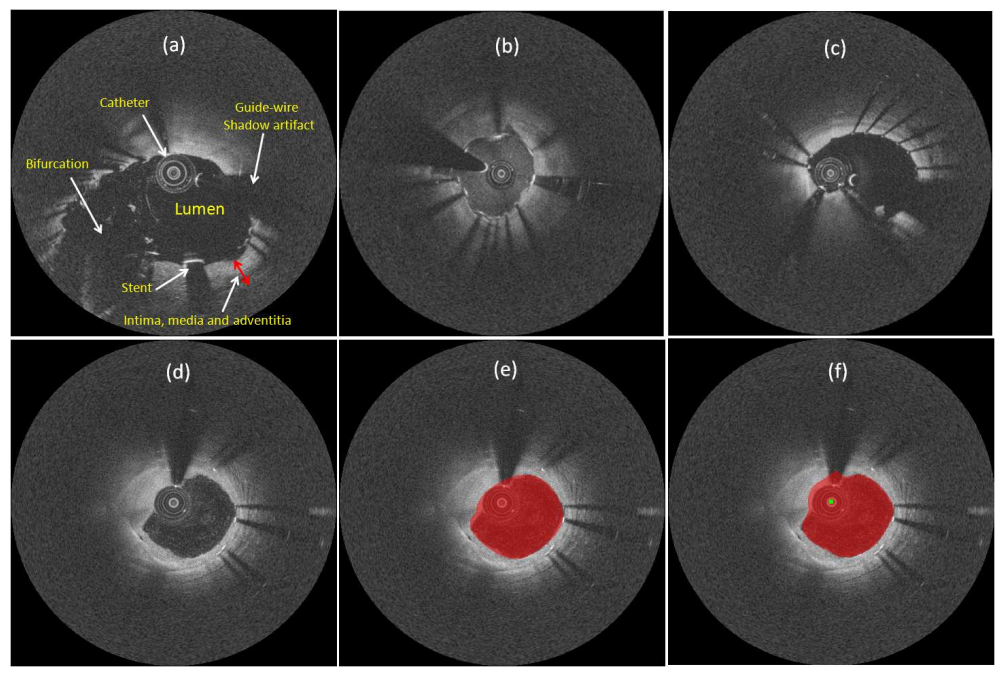

Figure 1: (a-c) Various complexities in OCT, (d-f) a segmentation example: original image (d), ground truth (e) and the result of the proposed method (f).

manual interference and improve segmentation accuracy. One of the early attempts is to apply an elliptical shape prior [18] to iteratively improve object segmentation. Then, a blob-like prior is used to extract the objects $[19,20]$. However, the specific shape assumption used in these methods may limit their applications. Some shape-free priors, such as connectivity prior [21], can be employed to avoid over-constraint on the overall shape. In addition, registration is often necessary [21]. Recently, a generic shape assumption, the star shape prior [22], is proposed in order to achieve robust performance with minimal user interaction (single user input for star centre). However, a heuristic balloon force is necessary in the cost function to avoid bias towards small segments, which is difficult to control. For lumen identification in OCT, shape prior knowledge has shown to provide useful geometric information of lumen area for reducing artefact interference [23, 24, 25]. 
In this paper, a novel shape prior based approach is proposed to address lumen segmentation, where a similar assumption to star shape prior used in [22] is implicitly imposed by unravelling the image in the polar coordinate system. Fig. 1(d-f) presents a segmentation example using the proposed method, where Fig. 1(e) is the ground truth and Fig. 1(f) shows the resulting image. This paper extends the preliminary work in [26], by applying our proposed method to OCT data and by providing substantial evaluation with comparison to several traditional techniques [22, 27, 28, 29]. The main contributions of this paper include the following aspects.

1. Instead of constructing the graph in the Cartesian coordinates, the image is transformed into a polar coordinate system and a graph is accordingly constructed. Therefore, the star shape prior is interpreted as the assumption that object boundary is unfolded in the polar coordinate system. By doing so, the bias towards short path is removed. This transformation is also applied to construct the optimal surface [30] where global minimum is achieved without user input.

2. A recently proposed image feature [31] is incorporated into the cost function instead of image intensity or local image gradient used in the conventional methods. This image feature, deriving from the gradient vector interaction over the whole image domain, can behave like a regional feature in segmentation. Another benefit with this feature used in our method is that a diffusion scheme can be applied to the derived vector field for dealing with image noise.

3. The generated image feature is applied to construct a node-weighted directed graph so that lumen segmentation can be obtained through the 
graph cut algorithm with the derived cost function. In OCT images, the centre of catheter area is the centre of star shape prior that is implicitly used in our method. Thus, our lumen segmentation is fully automatic without any user intervention.

4. The implicit use of star prior in the solution scheme allows the proposed method to effectively tackle the artefact interferences from the imaging environment such as catheter, stent and guide-wire. The experimental results demonstrate that our method performs comprehensively better than star graph cut [22] in both natural and OCT images. We also show comparisons to deformable-model based methods applied in OCT, such as the Chan-Vese model [29, 32], Vector Field Convolution (VFC) [27] and Distance Regularized Level Set Evolution (DRLSE) [28]. Moreover, one of the most recent data-driven based method, Mask RCNN [33], is employed to compare with the proposed method.

The rest of the paper is organised as following. In Section 2, the proposed method is described in detail, including pre- and post-processing, feature extraction, graph construction and minimisation with the proposed cost function. Section 3 presents the experimental results with evaluation of segmentation accuracy. The performance and relevant issues of the proposed method are discussed in Section 4. Finally, concluding remarks are provided in Section 5.

\section{Method}

In the proposed method, lumen segmentation in OCT is converted into searching a minimum $s-t$ cut in a derived arc-weighted digraph. We perform 
the preprocessing of the OCT images by transforming them from the Cartesian coordinates to a polar coordinate system and converting the catheter region to a homogeneous area with a given radius and constant intensity. A gradient convolution field (GCF) [31] is then employed to calculate the image feature, which makes use of appearance information obtained from both magnitude and direction of image gradients. For dealing with noise, the GCF field is refined by using a Laplacian diffusion scheme. Then, a directed graph is constructed to represent the image to be segmented and the graph nodal weight is calculated based on the derived features. Thus, lumen segmentation is converted into finding the minimum closed set as a height field segmentation is established in the polar coordinate system. Finally, a radial basis function $(\mathrm{RBF})$ based interpolation is applied to smooth out the interface oscillation and the OCT image is transformed back to Cartesian coordinates. Fig. 2 illustrates the main components and outputs of the proposed method in a flowchart, which are elaborated in the following subsections.

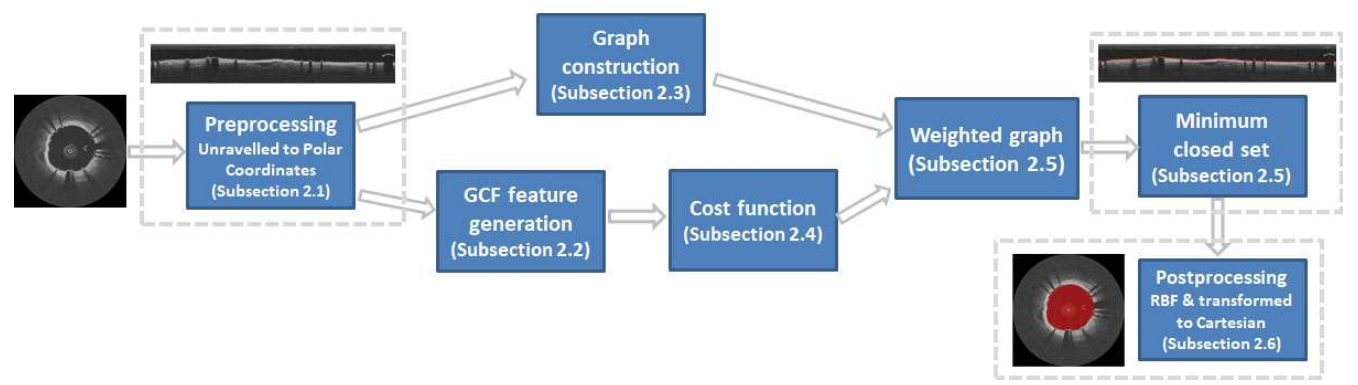

Figure 2: The flowchart with the main components and outputs of the proposed method. 


\subsection{Pre-processing}

Two steps of the preprocessing are necessary to facilitate our segmentation with prior and avoid bias towards shorter cut. The first one is the transformation of images from Cartesian coordinates to polar coordinates. The image is unraveled from the original Cartesian coordinates to polar coordinates based on the centre of the catheter. We assume that the object boundary intersects with each column of pixels once and once only, which is equivalent to the star shape assumption [22] that object boundary does not occlude itself from the catheter centre in Cartesian coordinates. A major benefit from this preprocessing is that it eliminates the need for a hand-crafted penalty term in the cost function to penalize bias towards shorter cuts. It also provides a regular graph grid that is convenient to work with, in contrast to the nonuniform graph structure in star graph cut method [22]. We adopt the optimal surface method, proposed in [30], to construct the graph in order to search for global solutions. Furthermore, the post-processing such as RBF smoothing can be more efficiently carried out since the 1-D interpolation will suffice in the polar coordinate system instead of the 2-D interpolation in the Cartesian coordinate system. The second preprocessing step, based on the transformed image, is to replace the catheter region with a homogeneous one by using a given radius. This is trivial process since the size of catheter is known beforehand and fixed in the image acquisition procedure.

\subsection{Image feature}

Fundamental to graph cut is the formulation of cost function, which can be generally categorised as edge based and region based approaches. Edge based cost functions assume that object boundary is largely collocated with 
image intensity discontinuity and typically use derivatives of image intensity function as a local estimation of likelihood of an object boundary, while region based ones are usually based on regional shape, intensity or texture information, e.g. piecewise constant assumption. Among these cost functions, image intensity values are often directly used for general image segmentation. Although graph cut algorithms can provide global optimum in two-level segmentation, a reliable but also generic image feature that does not assume strong image prior is desirable for segmentation that is useful for, for instance, object recognition. Intensity discontinuity is usually considered as the least constrained and most widely applicable object boundary estimation. However, its performance can be compromised by image noise, smooth varying intensity at object boundary, and so on. A major reason is that the generated feature used in graph cut algorithms is a local measurement without taking into account interactions among image gradient vectors. For example, a region with relatively large image gradient magnitude by varying gradient directions suggests that it is unlikely a location of object boundary, despite their large magnitude. In contrast, weak gradient vectors that are aligned to each other suggest greater likelihood of object boundary than what the magnitude itself suggests. Very recently, we proposed a gradient convolution field (GCF) [31] that is a result of global gradient interactions across the whole image domain. In this work, we show that its divergence can be used as a reliable image feature for graph cut.

For convenience, $\nabla_{i} I=f \hat{I}_{x}$ and $\nabla_{j} I=f \hat{I}_{y}$ denote the two components of the image gradient $\nabla I$ in the image coordinates $(i, j)$, respectively, i.e. $\nabla I=\left(\nabla_{i} I, \nabla_{j} I\right)^{T}$ where $f$ is edge map (magnitude). As introduced in [31], 
$\mathrm{GCF}$, i.e. $\mathbf{E}=\left(E_{i}, E_{j}\right)$, is obtained by the following equations:

$$
\left\{\begin{array}{l}
E_{i}(\mathbf{x})=\nabla_{i} I * k(\mathbf{x})=\sum_{\mathbf{s} \neq \mathbf{x}} \frac{\nabla_{i} I(\mathbf{s})}{R_{\mathbf{x s}}}=\sum_{\mathbf{s} \neq \mathbf{x}} f(\mathbf{s}) \frac{\hat{I}_{x}(\mathbf{s})}{R_{\mathbf{x s}}}, \\
E_{j}(\mathbf{x})=\nabla_{j} I * k(\mathbf{x})=\sum_{\mathbf{s} \neq \mathbf{x}} \frac{\nabla_{j} I(\mathbf{s})}{R_{\mathbf{x s}}}=\sum_{\mathbf{s} \neq \mathbf{x}} f(\mathbf{s}) \frac{\hat{I}_{y}(\mathbf{s})}{R_{\mathbf{x s}}},
\end{array}\right.
$$

where the kernel $k(\mathbf{x})=m(\mathbf{x}), R_{\mathbf{x s}}$ denotes the distance from $x$ to $s$ and we choose the magnitude function $m$ as an inverse of distance, i.e. $m(r)=$ $1 / r^{\zeta}$ with $\zeta=1$. Since we further compute the spatial derivatives of the convolution results, the spatial decay is actually raised to power of two, i.e. $\zeta=2$. The convolution computation is across the whole image domain in (1) and both the magnitude and direction information of image gradient take part in the GCF calculation. Thus, the original image gradient vectors have extended their influence from immediate vicinity of edge pixels to much larger neighbourhood. The gradient convolution divergence can be obtained by calculating the change rate of $\mathrm{GCF}$, i.e.

$$
B=\nabla \cdot \mathbf{E}(\mathbf{x})=\nabla \cdot\left(E_{i}, E_{j}\right)
$$

In fact, this GCF divergence has an intrinsic link to the magnetic field used in the MAC model [34] in a variational framework. Specifically, when $\zeta=1$, $B$ is equivalent to the third and only effective component of the magnetic field in the MAC model. Hence, the positive and negative values of this GCF divergence indicate the intensity discontinuities in the image, which can be used to differentiate foreground with background. The zero crossings of the value would therefore identify the location of object boundaries. This proposed GCF based feature is thus a generalisation of the effective component used in MAC. In addition, we can refine this feature by diffusing the GCF field to overcome, for instance, noise interference. 
It is worth noting that the GCF is distributed along the edge direction and thus appropriate diffusion in its components can help enhance the GCF magnitude along the edge and produce better boundary description. There are various diffusion strategies for this smoothing task. For implementation convenience and less parameter tuning, we use an isotropic (Laplacian) diffusion scheme in this paper, which is carried out by solving the following Euler equations.

$$
\left\{\begin{array}{l}
\frac{\partial}{\partial t} \mathcal{E}_{i}(\mathbf{t}, \mathbf{x})=p\left(E_{i}\right) \Delta \mathcal{E}_{i}(\mathbf{t}, \mathbf{x})-q\left(E_{i}\right)\left(\mathcal{E}_{i}(\mathbf{t}, \mathbf{x})-E_{i}\right), \\
\frac{\partial}{\partial t} \mathcal{E}_{j}(\mathbf{t}, \mathbf{x})=p\left(E_{j}\right) \Delta \mathcal{E}_{j}(\mathbf{t}, \mathbf{x})-q\left(E_{j}\right)\left(\mathcal{E}_{j}(\mathbf{t}, \mathbf{x})-E_{j}\right),
\end{array}\right.
$$

where $\Delta$ is the Laplacian operator, $\mathcal{E}_{i}(0, \mathbf{x})=E_{i}(\mathbf{x}), \mathcal{E}_{j}(0, \mathbf{x})=E_{j}(\mathbf{x})$, and $p(y)$ and $q(y)$ are given as:

$$
p(y)=\exp (-|y| / K), q(y)=1-p(y),
$$

where $K$ is a constant.

Due to the nature of the isotropic diffusion, the Laplacian diffusion behaves the same regardless of the diffusion direction. This can reduce the interference of noises, especially Gaussian noise, to some extent and therefore help present the geometric properties more effective. Fig. 3 illustrates an example by applying Laplacian diffusion to the GCF of a noisy image. Comparing to Fig. 3 (b), the visualisation of the GCF divergence $B$ in Fig. 3 (c) shows that the intensity discontinuities on the boundary become more significant after the diffusion. This improvement of the GCF divergence $B$ actually comes from the enhancement of GCF by applying the Laplacian diffusion. Fig. 3 (e) illustrates that the GCF in the red region in Fig. 3 (a) gets enhanced after the diffusion. 


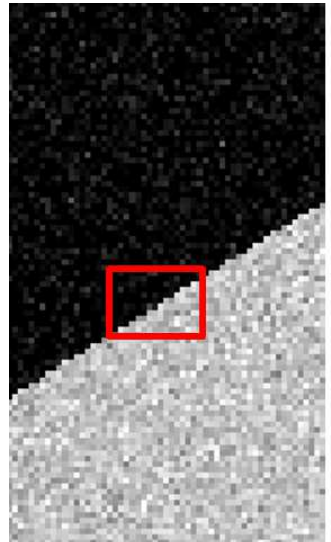

(a) Noise image

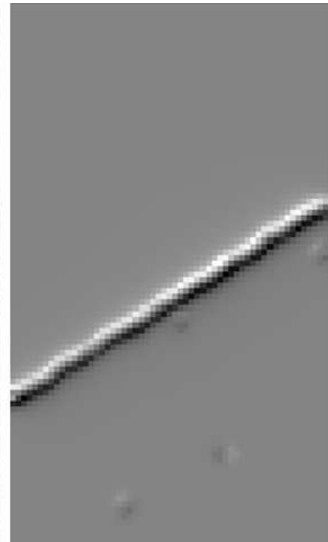

(b) $B$ before diffusion

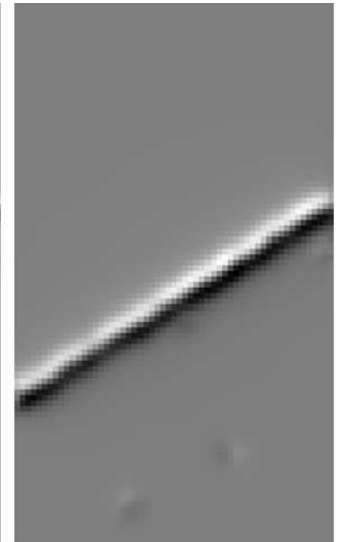

(c) $B$ after diffusion

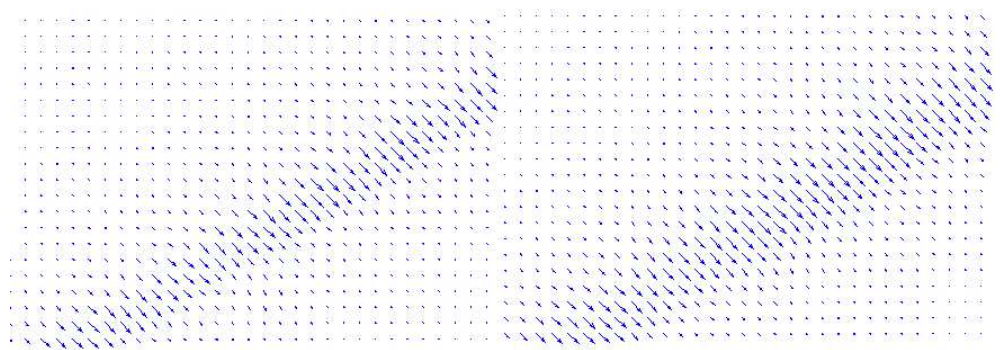

(d) GCF before diffusion

(e) GCF after diffusion

Figure 3: (a) Image with Gaussian noise, (b) GCF divergence (B) before diffusion, (c) GCF divergence $(B)$ after diffusion, (d) GCF in the red region before diffusion, (e) GCF in the red region after diffusion. 


\subsection{Graph construction}

To perform the segmentation, a directed graph is constructed and lumen segmentation is then transformed into searching a minimum closed set in the derived node-weighted directed graph. A closed set $\mathbb{C}$ is defined as a subset of nodes in a directed graph that all successors of any nodes in $\mathbb{C}$ belong to $\mathbb{C}$ as well. The aggregated cost of nodes in a closed set is the cost of the set. In a weighted directed graph, searching a minimum closed set is in effect a minimum $s-t$ cut problem that is polynomial complexity [35].

The directed graph $G=<V, E>$ is constructed as follows. Each node $V(x, y)$ corresponds to a pixel in 2-D image $I(x, y)$ in polar coordinates. Two types of arcs, intra-column arcs and inter-column ones, are included in the graph $G$. Every node $V(x, y)$ in intra-column, where $y>0$, has a directed arc to the node $V(x, y-1)$. Meanwhile, each node $V(x, y)$ in intercolumn links to a node $V(x+1, \max (0, y-\Delta))$ with a directed arc, where $\Delta$ is a smoothing factor ( $\Delta=3$ used for this study) and its purpose is to guarantee graph connectivity. Similarly, node $V(x+1, y)$ is established to connect $V(x, \max (0, y-\Delta))$. For keeping a closed graph, the last row of the graph is to connect each other of the nodes. Fig. 4 illustrates this directed graph on two adjacent columns. Fig. 5(c-d) presents the unravelled images corresponding to Figs. 5 (a) and (b), where the left image is the original one and the right image is with the resulting segmentation using our method. The cost from the image, detailed in Subsection 2.4, is then assigned to the nodes of this directed graph, which can be transformed to an edge weighted graph with the upper interface of its minimum closed set from bottom of the graph corresponds to an optimal cut. 


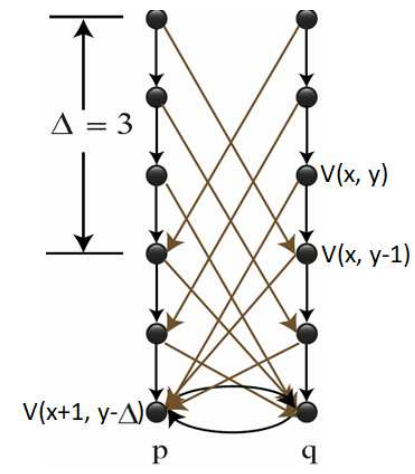

Figure 4: An illustration of inter-column and intra-column arcs.

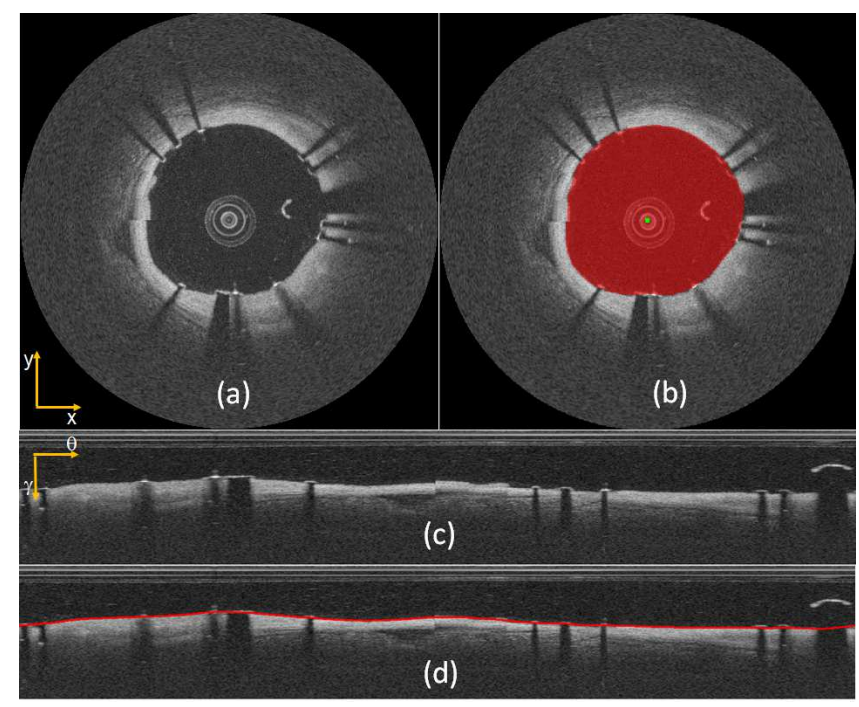

Figure 5: Original image (Number 13) (a), Resulting image (b), Unravelled image in the polar coordinate system (c) and segmentation result in the polar coordinate system (d). 


\subsection{GCF-based cost function}

In the derived directed graph, each node represents a pixel in the image while the arcs between pixels are described by the connectivity of the pixels that certain constraints of smoothness and interaction are satisfied. For segmenting the image, cost function is designed to calculate the cost of the cut that is the sum of costs of all arcs from $S$ to $T$ where $(S, T)$ is a partition of nodes in the derived graph into two disjoint sets $S$ and $T$. The minimiser of cost function is the minimum-cut of the graph that the minimal cost closed set can be computed accordingly, which results in the lumen segmentation of the OCT image. Thus, the appropriate cost function can help greatly improve the performance of the graph-based segmentation method used. In general, the information to take into account in the designing of cost function comes from either edge-based or region-based attributes of the image to be segmented.

By considering the relationship between edge information and lumen border, edge-based cost function is used in our approach. The cost $C(x, y)$ assigned to each node $V(x, y)$ in the directed graph is derived with respect to image features described in Subsection 2.2. The cost function indicates the likelihood of each node in the graph representing the desired interface.

The cost of a cut in $G$ is described as $\mathbb{E}=\sum_{V \in S} C(x, y)$, where $C(x, y)$ denotes the cost function and $S$ is a path in the derived directed graph $G$. As discussed in Subsection 2.2, the zero-crossings of the GCF based feature computed from gradient vector interaction indicate the location of object boundary. Moreover, the degree of the feature oscillation at the zero-crossing suggests the strength of object boundary. However, a direct assignment of 
(a) Before diffusion

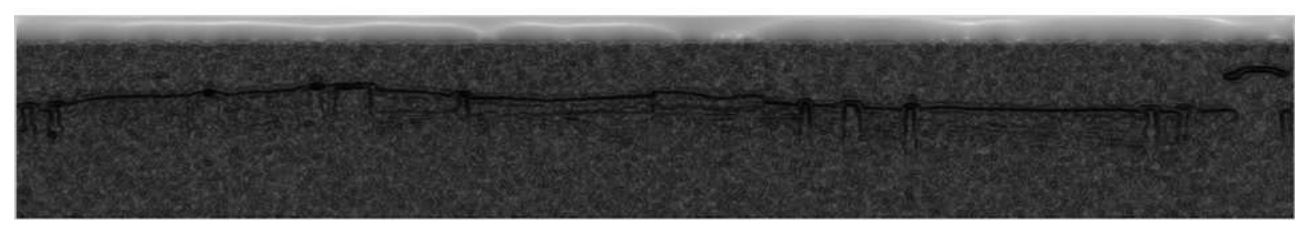

(b) After diffusion

Figure 6: A visual example of the cost term generated in Eq. (5) on the image of Number 13. (a) shows the cost term before the Laplacian diffusion Eq. (3). (b) shows the cost term after the Laplacian diffusion.

the GCF based feature to the graph as nodal cost would be inappropriate, since the minimum or maximum of this feature is not an indication of either location or strength of object boundary. Thus, without loss of generality, a simple transformation can be applied. Due to the decay of the GCF based feature from object boundary being exponential (cf. Eqs. (1) and (2)), a log transformation is used to avoid extreme values to be assigned to the graph. The cost function is thus formulated as follows:

$$
C(x, y)=-\log |\nabla \cdot \mathbf{E}(\mathbf{x})|=-\log |B|
$$

where $\mathbf{E}(\mathbf{x})$ is GCF that is defined in Eqs. (1). A visual example of this cost term is shown in Fig. 6.

\subsection{Graph cuts and minimum closed set}

The segmentation of lumen can then be implemented by obtaining the minimum cut on the constructed graph. Each node is weighted by the cost 
of the corresponding pixel in the image. The weight of the node is a value representing its rank that will transform the segmentation problem into the calculation of a minimum closed set. In order to use the max-flow min-cut algorithm to perform the cut, the weight of each graph node is transformed as follows:

$$
\omega(x, y)=\left\{\begin{array}{lr}
C(x, y) & \text { if } y=0, \\
C(x, y)-C(x, y-1) & \text { otherwise. }
\end{array}\right.
$$

where $C$ is the cost function and $\omega$ the weight for each node in the directed graph. The weighted nodes can be further decomposed into nonnegative and negative sets with respect to the weight values obtained according to Eq. (6). These generated weights are used to construct an edge weighted graph. In this constructed graph, the negative weights (using their absolute value) are assigned to the edges between these nodes and an assumed source $s$, and the positive weights go to the edges between these nodes and an assumed sink $t$. The edges between the negative nodes and positive nodes are defined with infinity cost that enforce the smoothness and close set constraints in the constructed graph. The interface corresponding to the object boundary is produced as the minimum closed set segments from the graph using $s-t$ cut. Fig. 5(d) shows an example of segmentation in polar coordinates and its result recovered in Cartesian coordinates is given in Fig. 5(b).

\subsection{Post-processing}

Although a smoothing constraint is used in graph construction to minimize sudden changes in the interfaces, local oscillations in the segmented lumen may still appear. Here, we apply an RBF interpolation based on the 


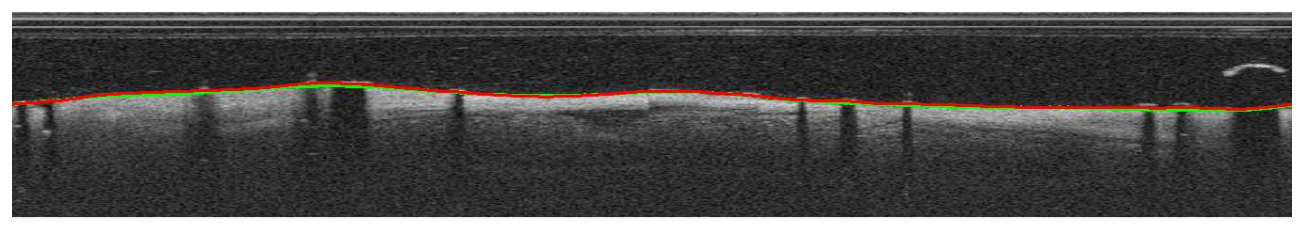

Figure 7: Illustration of the RBF interpolation on the resulting segmentation (the image is the same in Fig. 5). The green line is the result before the interpolation and the red line is after the interpolation.

thin plate function to eliminate the interface oscillation and obtain more accurate interfaces. The RBF interpolation is formulated as follows.

$$
f(x)=c_{0}+c_{1} x+\sum_{i=1}^{n} \lambda_{i} \phi\left(\left|x-x_{i}\right|\right)
$$

where $c_{0}, c_{1}$ and $\lambda_{i}$ are coefficients, $x_{i}$ is the input node, the RBF function is $\phi(r)=\phi\left(\left|x-x_{i}\right|\right)$, where $\phi(r)=r^{2} \log (r+1)$.

In this study, the RBF processing is carried out in $1-D$ because the images are represented in polar coordinates. Fig. 7 illustrates the performance of this RBF interpolation (red line) in the resulting segmentation (green line).

\section{Experimental results}

In order to validate the performance of the proposed method in vessel lumen border segmentation, a total of 2283 frames from 10 in vivo pullbacks of human subjects were acquired with a Frequency Domain OCT Imaging System (C7-XR, LightLab) and were then used for the analysis in this study. In addition, these image datasets were captured from both left anterior descending arteries (LAD) and right coronary arteries (RCA), containing various forms of artefacts such as fibrous plaque, calcification, stent, and bifurcation. To establish the ground truth, these 2283 images were manually 
labelled for identifying the lumen area under the supervision of a radiologist in Singleton Hospital, Swansea, UK.

For comparison, five methods are employed to compare with the performance of the proposed method. Among these methods, the modified ChanVese model [32, 29] is a typical region-based technique but utilises a Gaussian filtering process to keep the signed distance function (SDF) condition during curve propagation so as to avoid re-initialisation; DRLSE [28] is an edgebased method without reinitialisation by minimising an energy functional with a distance regularisation term; VFC [27] is a derived deformable model by proposing vector field convolution as a new external force for active contours; star graph cut [22] is a recently proposed graph based method by incorporating a star shape prior into the graph cut formulation; and Mask RCNN [33] is the most recent CNN model for instance segmentation.

We compare our method to star graph cut quantitatively and qualitatively with all the acquired data, since star graph cut is the closest to our method. In addition, a series of natural images are used to qualitatively compare our method with star graph cut. For other three deformable methods used in comparison, they are all initialization-dependent and are based on continuous optimisation. We need to choose appropriate iteration number for best results while an appropriate initialisation is carefully set in the experiments. With these considerations, we randomly select 226 images from the total 2283 OCT images for demonstrating their performances. For Mask RCNN, the same set of 226 images is used to evaluate its performance. The rest 2057 images of the total 2283 images are used to train the model for lumen detection, with the ratios of $7: 2: 1$ for training, testing and validation respectively. Labelme 
[36] is employed to annotate the data.

\subsection{Parameter setting}

In the proposed method, there are a number of parameters used at the relevant stages. The diffusion parameter in feature extraction is fixed to 0.05 and the value for $\Delta$ in graph construction is set as 3 . The RBF coefficients in postprocessing are obtained by satisfying the interpolation constraints, the border points which define that the RBF centres must interpolate the function $y_{i}=f\left(x_{i}\right)$. Therefore, these parameters can be calculated with a linear system because the RBF is a linear equation with respect to these unknown parameters.

\subsection{Results on real OCT data}

Fig. 8 presents typical results obtained using star graph cut (Column (c)) and the proposed method (Column (d)). The original images and their ground-truth are shown in Columns (a) and (b). Comparing to the ground truth, in most of the cases star graph cut over-segmented the images due to its use of the balloon force. In contrast, our method performs well in handling the interference of various artefacts. For examples, the images in Row 7 show that the segmentation of star graph cut is severely interfered due to the artifacts of bifurcation and guide-wire shadow. In Row 9, the guide-wire shadow artifact severely affects the performance of star graph cut but our method accurately identified the lumen area. The stent artifacts of the image in the last row cause the over-segmentation in star graph cut. All of these results are visually evident that the proposed method performs better than star graph cut when dealing with common artefacts in OCT. 
To quantitatively evaluate the performance, we employ the following six metrics: Absolute Mean Difference (AMD), Hausdorff Distance (HD), Area Overlap (AO), sensitivity (precision), specificity (recall) and $F_{1}$ score (the harmonic average of the precision and recall). For AMD and HD, the performance is considered better if the values of these metrics are smaller. For AO, sensitivity, specificity and $F_{1}$ score, the larger the better. The quantitative results are presented in Table 1. The proposed method outperforms star graph cut in all metrics except for sensitivity. The sensitivity for the star graph cut is slightly higher than ours because star graph cut tends to over-segment the image. The results of $F_{1}$ score clearly show that our method outperforms star graph cut. For checking the difference between our approach and star graph cut, the paired t-test is conducted on 2283 images with consideration of the hypothesis that there is no significant difference in the mean of the overlap-to-GT of all images at the significance level $\alpha=0.001$, i.e.

$$
H_{0}: \mu_{1}=\mu_{2}, H_{1}: \mu_{1} \neq \mu_{2}
$$

where $\mu_{1}$ is the mean of the overlap to the ground truth obtained by our method, $\mu_{2}$ is the overlap mean for the star graph cut. Table 2 presents the testing results. For $\alpha=0.001$ and 2282 degrees of freedom, the critical value is 3.291. Since $6.16>3.291, H_{0}$ is rejected and this indicates that there is significant difference between our approach and the star graph cut.

In cases where the lumen hardness is severely disrupted, the performance of the proposed method can be adversely affected. Such examples are presented in Fig. 9. In Rows 1 and 2, the results show that the bifurcation leads to the poor performance. The stent artefacts also can cause undersegmentation for our method as shown in Rows 3, 4 and 5. 
Table 1: Quantitative results with 2283 images for star graph cut [22] and the proposed. AMD: Absolute Mean Difference(pixel); HD: Hausdorff Distance(pixel); AO: Area Overlap(\%); Sensitivity(\%); Specificity(\%); $F_{1}$ Score(\%).

\begin{tabular}{|c|c|c|c|c|c|c|}
\hline & AMD & HD & AO & Sensitivity & Specificity & $F_{1}$ Score \\
\hline $\begin{array}{c}\text { Star graph cut } \\
{[15]}\end{array}$ & 5.85 & 22.11 & 91.27 & 96.52 & 98.07 & 97.29 \\
\hline Proposed & 4.94 & 19.09 & 92.51 & 96.03 & 98.85 & 97.42 \\
\hline
\end{tabular}

Table 2: Paired t-test for testing the difference between our method and the star graph cut.

\begin{tabular}{|c|c|c|c|}
\hline Difference mean & Std. deviation & Std. error mean & t-statistics \\
\hline 1251.24 & 9703.15 & 203.08 & 6.16 \\
\hline
\end{tabular}


(a) Original

(b) Ground-truth (c) Star graph cut (d) Proposed
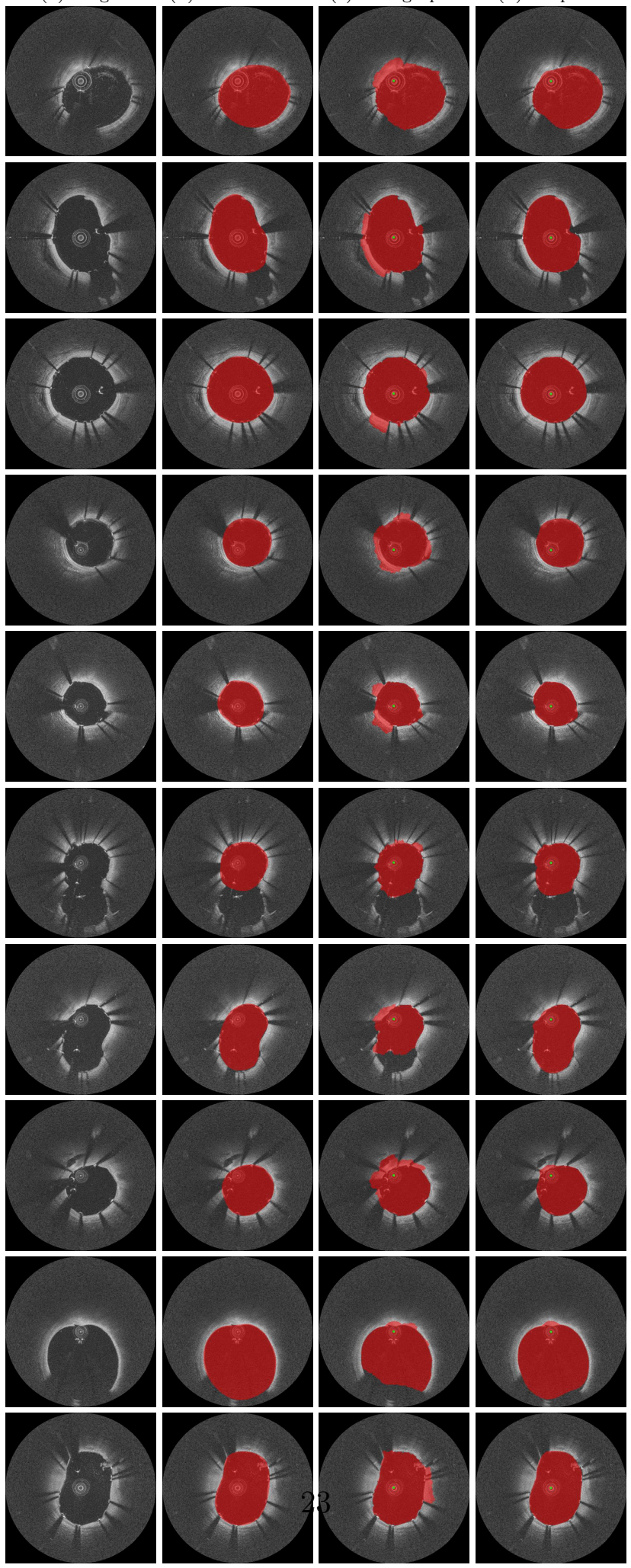

Figure 8: Comparison with star graph cut [22]. Column (a): Original image, Column (b): Ground-truth, Column (c): Star graph cut, Column (d): Proposed method. 


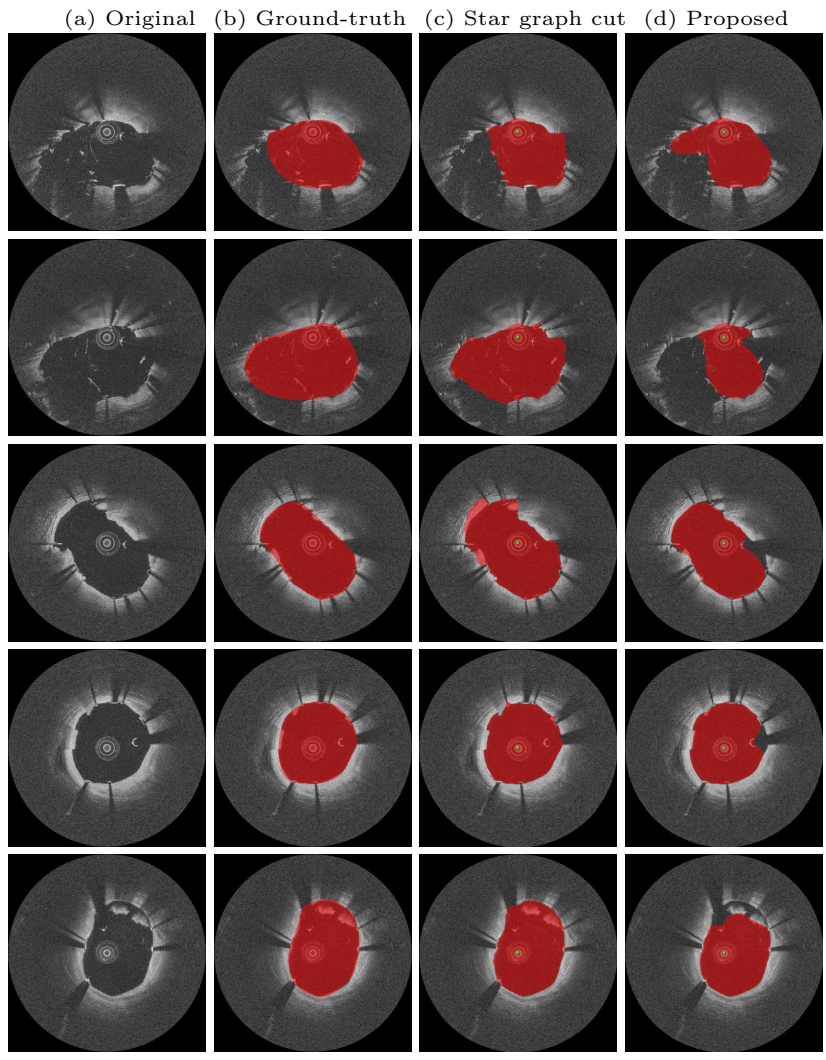

Figure 9: Cases with the inferior performance of our method. Column (a): Original image, Column (b): Ground-truth, Column (c): Star graph cut, Column (d): Proposed method. 
Table 3 shows the quantitative results of three deformable-model based methods and Mask RCNN, along with star graph cut and our method. The proposed method outperforms significantly over these methods. The ChanVese method can generally performed better than the edge-based methods. However, it did not well cope with the artifacts in the OCT images. Its AMD is measured at 39.99 and the AO is merely $38.67 \%$. Due to the use of vector field feature, VFC performed slightly better with the AMD measured at 14.34 and the HD reaches 50.03. DRLSE was not able to detect the lumen properly because it is directly based on the edge information. Mask $\mathrm{RCNN}$ is a data-driven method that may lead to some feature extraction issues such as underfitting or overfitting. The evaluation results show that its performance is inferior to our method in terms. The paired t-test is also applied to these methods to demonstrate the difference between our method and others. Table 4 shows the generated values of the t-statistics that are all greater than the critical value 3.291 for the significance level $\alpha=0.001$. Thus, $H_{0}$ is rejected in Eg. (8) and the significant difference exists between our method and the others.

For visualising the performance, five resulting examples are presented in Fig. 10. In Column (c), due to the nature of the piece-wise formulation, the lumen area is segmented as the background while the bright area is detected for [29]. In contrast, VFC (Column (e)) can perform well in some cases but it has great difficulties in the situation of severe artifacts such as bifurcation in the last three cases. Column (d) shows that DRLSE has the similar performance to VFC. However, DRLSE's overall performance is poorer than VFC because it is sensitive to weak edges. As sown in Column (f), Mask 
Table 3: Quantitative results with 226 images for the methods in [29, 27, 28]. AMD: absolute mean difference(pixel); HD: Hausdorff distance(pixel); AO: area overlap(\%); Sensitivity $(\%)$; Specificity $(\%) ; F_{1} \operatorname{Score}(\%)$.

\begin{tabular}{|c|c|c|c|c|c|c|}
\hline & AMD & HD & AO & Sensitivity & Specificity & $F_{1}$ Score \\
\hline $\begin{array}{c}\text { Improved } \\
\text { Chan-Vese [29] }\end{array}$ & 39.99 & 95.77 & 38.67 & 38.78 & 99.86 & 55.87 \\
\hline VFC [27] & 14.34 & 50.03 & 80.05 & 85.65 & 97.67 & 91.27 \\
\hline DRLSE [28] & 28.44 & 62.75 & 68.02 & 94.72 & 86.57 & 90.46 \\
\hline $\begin{array}{c}\text { Mask RCNN } \\
{[33]}\end{array}$ & 6.54 & 54.47 & 82.84 & 94.98 & 96.85 & 95.91 \\
\hline Proposed & 4.61 & 17.08 & 92.75 & 96.37 & 98.83 & 97.59 \\
\hline
\end{tabular}

RCNN produced promising results. However, it tends to under-segment the lumen region.

\subsection{Results on natural images}

We further provide a comparison analysis on a series of natural images with various conditions. Fig. 11 presents the visual comparison of the proposed method against star graph cut. The original images are shown in the column (a), followed by the results of star graph cut (b) and the results of the proposed method (c). These test images show varying degrees of difficulties for object identification, such as weak edge ("cat", "train", "grain"), non-uniform intensity ("tiger", "white bear", "grain"), textured appearance ("pig", "train"), and cluttered background ("pig", "black bear", "tiger"). 
(a) Original (b) Ground-truth (c) Improved CV (d) DRLSE (e) VFC (f) Mask RCNN (g) Proposed
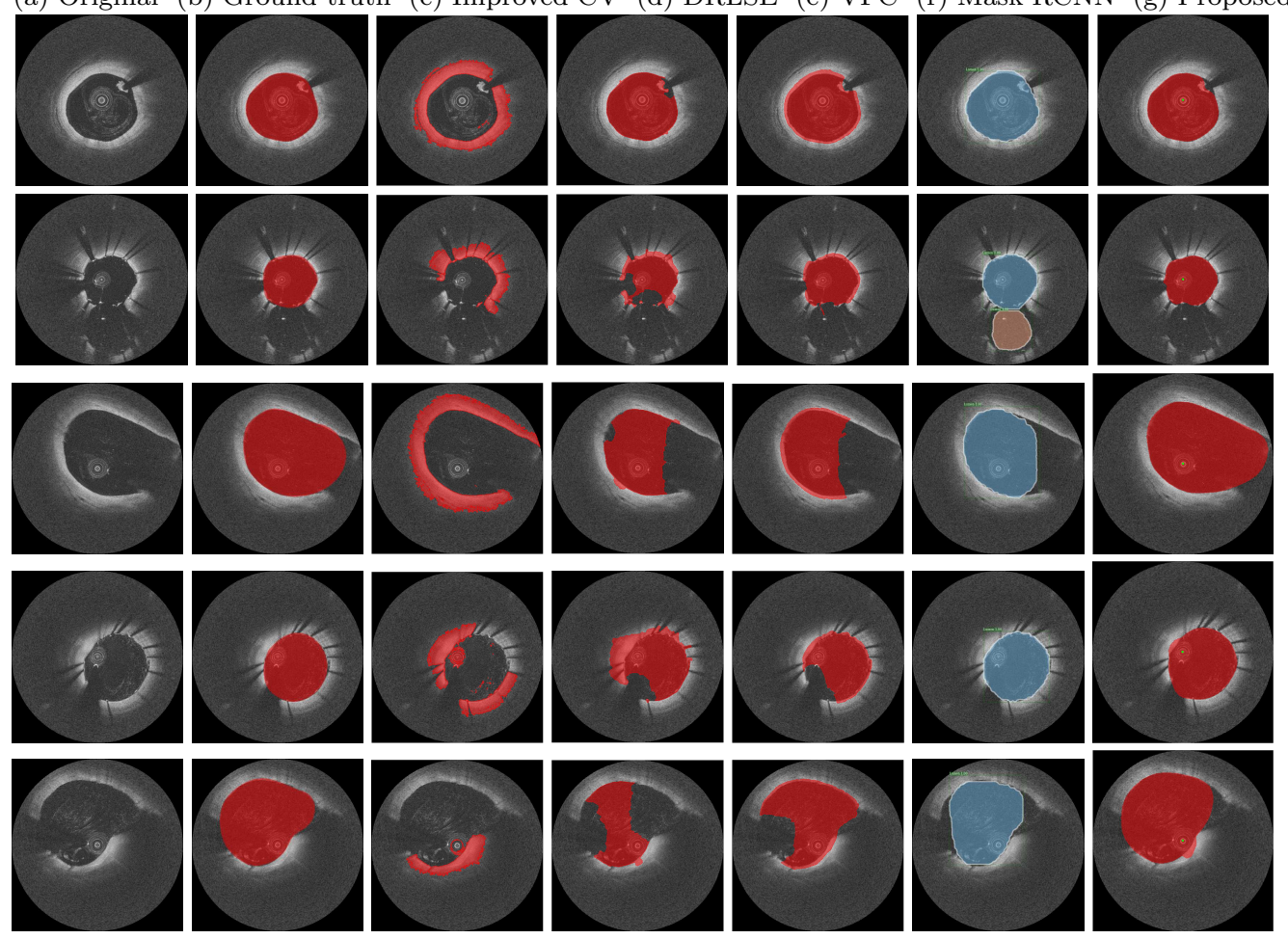

Figure 10: Results of the traditional methods in OCT. Column (a): Original image, Column (b): Ground-truth, Column (c): Improved Chan-Vese [29], Column (d): DRLSE [28], Column (e): VFC [27] and Column (f): Mask RCNN [33] (g): Proposed method. 
Table 4: Paired t-test for testing the difference between our method and other methods with 226 images.

\begin{tabular}{|c|c|c|c|c|}
\hline & $\begin{array}{c}\text { Difference } \\
\text { mean }\end{array}$ & $\begin{array}{c}\text { Std. } \\
\text { deviation }\end{array}$ & $\begin{array}{c}\text { Std. error } \\
\text { mean }\end{array}$ & t-statistics \\
\hline $\begin{array}{c}\text { Improved } \\
\text { Chan-Vese [29] }\end{array}$ & 50916.85 & 35033.21 & 2330.40 & 21.85 \\
\hline VFC [27] & 12271.62 & 22580.45 & 1502.37 & 8.17 \\
\hline DRLSE [28] & 33518.49 & 13944.09 & 927.53 & 36.14 \\
\hline Mask RCNN [33] & 7019.13 & 5264.52 & 350.19 & 20.05 \\
\hline
\end{tabular}

In the resulting images, the regions in red indicate the segmentation result. For star graph cut, the parameters are carefully tuned as suggested in [22] and the star centre (highlighted point) is carefully placed in order to achieve best results. However, star graph cut tends to under-segment objects in the presence of weak edges, such as "cat", "train" and "grain", and oversegment when images are cluttered, such as "pig", "black bear" and "tiger". As discussed in [22], this problem is in fact due to the use of the intractable "balloon" force that aims to deal with the bias issue in star shape prior. In contrast, the proposed method achieved consistently better performance with the same user initialisations (star centres, i.e. highlighted in green).

\section{Discussion}

\subsection{Handcrafted feature versus data driven feature}

The proposed approach aims to present a generic lumen segmentation method with the consideration for the OCT data characteristics. Although 
(a) Original Image
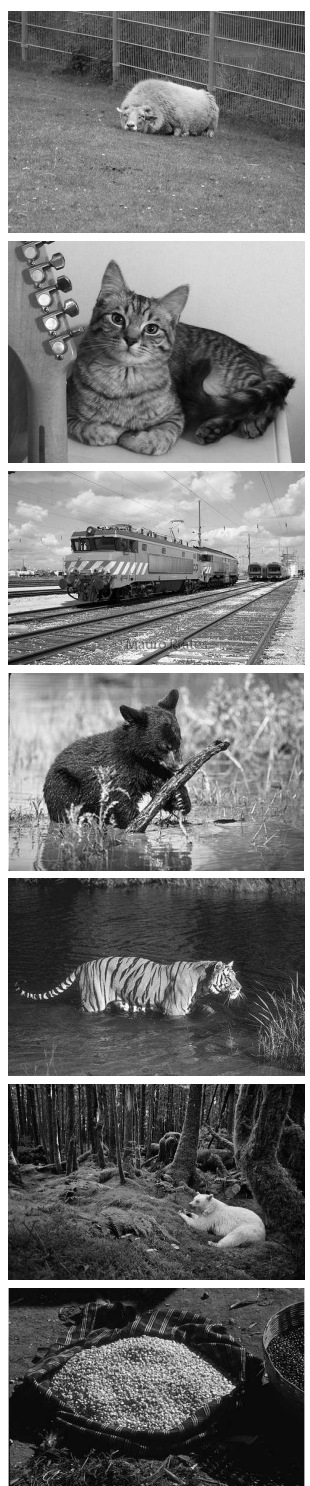

(b) Star graph cut
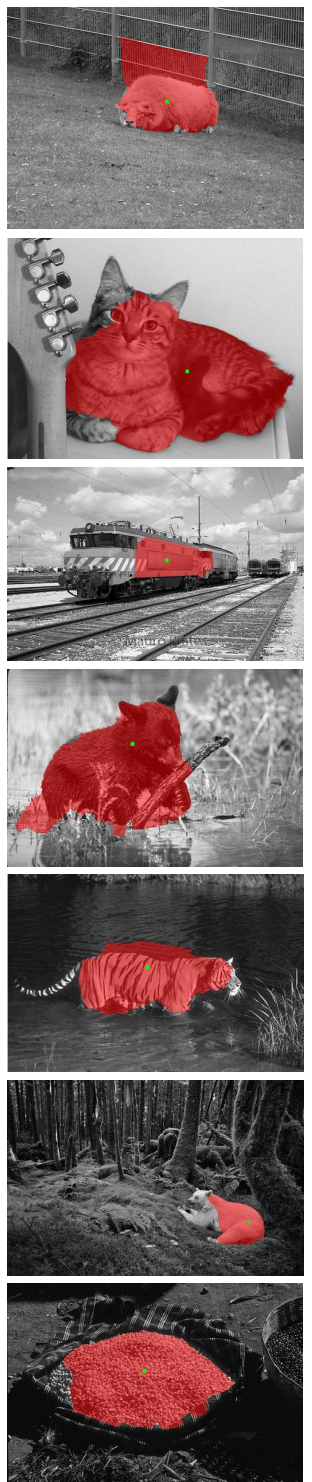

(c) Proposed Method
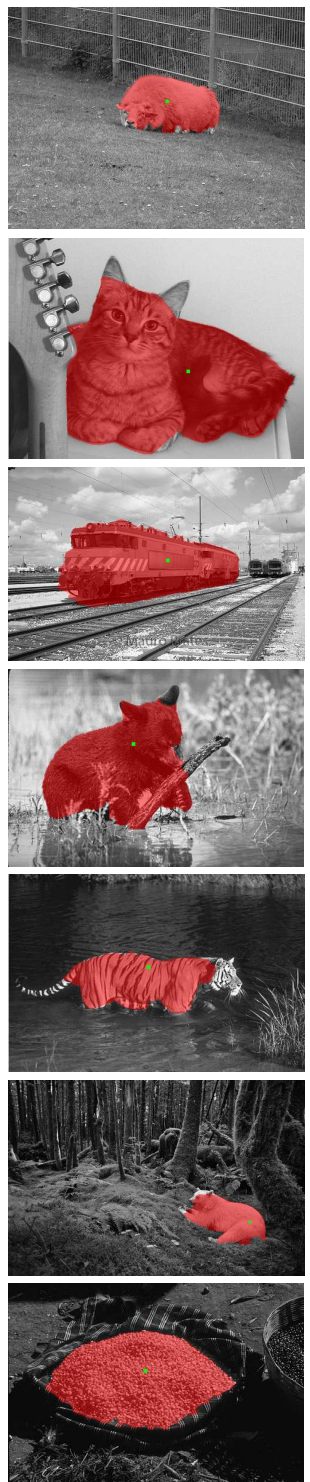

Figure 11: Comparison results for various images. Column (a): Original Image; Column (b): Results using star graph cut [15] ; Column (c): Results using the proposed method. The green dots indicate the user initializations. 
the feature extracted in our approach is handcrafted, such as GCF, the designed solution can be applied to any OCT based lumen data without the necessity of ground truths. The proposed approach has at least two advantages compared to deep learning based methods in dealing with lumen segmentation. The proposed method is fully automatic and can be easily adapted to different OCT data sets, contrary to data-driven methods that have considerable training and re-training overhead. The proposed method is also much more efficient and since it is unsupervised it does not require large amount of training data with ground-truth labelling. Furthermore, the proposed approach is not limited to segment OCT data. In fact, the experimental results in Fig. 11 demonstrate that the proposed approach is also capable of segmenting natural images. Our proposed method also integrates a shape prior and geometric constraints through the design of the directed graph, which are difficult for data driven models to implement.

In supervised learning, deep learning can take the advantage of data driven features over the traditional methods with the handcrafted features. However, its data oriented nature means that its performance is subject to specific dataset. Particularly, the deep learning based approaches are difficult to adapt to different datasets. For example, Mask RCNN can be used to segment the lumen area with the manual ground truth but this approach cannot be applied to detect the generic objects in natural images. In contrast, our proposed approach is capable of carrying out the segmentation for both the lumen and natural objects as shown in Fig. 11. 


\subsection{On GCF based cost function and the graph cut algorithms}

In our solution scheme, the GCF plays a crucial role to gain the advantages over the traditional methods. Firstly, the GCF computation takes into account both gradient direction and edge strength in the whole image domain, unlike the traditional methods that often just make use of edge strength such as VFC [27], DRLSE [28] or those merely based on local fittings such as Chan-Vese [29]. Secondly, GCF can be refined with the employed diffusion scheme that enables it to cope with excessive image artefact in OCT. Lastly, unlike the traditional methods, our generated GCF is not directly used in the minimization scheme. Alternatively, the GCF is employed to generate the cost function in the constructed graph and then the graph cut algorithms are applied to find the optimal solution.

The similarities between our method and star graph cut [22] come to two folds. One is that both methods search for a solution via the graph model, which is based on the graph cut algorithm. The other is that the star shape prior is used in both methods for achieving robust performance. The differences are in the solution schemes, which are threefold. Firstly, our approach applies the graph model in polar coordinates rather Cartesian coordinates for star graph cut. Secondly, the cost function in our approach employs the proposed GCF feature that is able to avoid the bias issue due to a heuristic balloon force used in star graph cut. Thirdly, our approach is capable of applying diffusion techniques to improve feature extraction and cost function in order to obtain better performance.

The higher sensitivity indicates the star graph cut is with more ability to correctly detect the foreground pixels. However, star graph cut has much 
lower specificity that means it over segment the foreground regions, which leads to poorer results. Sensitivity alone could not determine superiority of a method. Considering all metrics together, the proposed method clearly outperforms the star graph cut.

\subsection{Computational complexity}

As shown in Fig. 2, the proposed approach mainly consists of feature extraction $(\mathrm{GCF})$, graph construction and minimization scheme i.e. minimum closed set (MCS). For an image $(N \times N)$, the calculation of the GCF is performed with fast Fourier transform (FFT) in the frequency domain. The computational complexity is $O\left(N^{2} \log (N)\right)$ [31]. For MCS detection, the Boykov-Kolmogorov algorithm [16] is implemented, which the computational complexity is $O\left(N^{4} \times M\right)$ ( $M$ is the number of arcs in the constructed graph). Thus, the computational complexity of our proposed approach is $O\left(N^{4} \times M\right)$. All experiments were conducted on a standard i7-6700 3.4Ghz workstation with 32 GB of memory. The average execution time on the test dataset of 226 images is $0.997210 \mathrm{~s} / \mathrm{f}$.

\section{Conclusion}

This paper presents a graph based segmentation without any user interaction in OCT. For making use of image information efficiently, a region-like image feature is used to generate the cost function, which is derived from global interactions of gradient vectors across the whole image domain. We unravel images into the polar coordinate system and the corresponding graph for the lumen segmentation is constructed in the 2-D polar space so that the 
issue of short cut is tackled and also the star shape prior is implicitly incorporated into the solution scheme. Furthermore, for enhancing the ability of coping with artefact interference in our method, Laplacian diffusion is employed to refine image feature. Experimental results in OCT demonstrated promising performances in comparison with several alternative methods. It is evident that the proposed method performed much better against the traditional deformable-model based methods and the data driven deep learning method. The focus of this work is $2-d$ lumen segmentation. More sophisticated diffusion may be applied to GCF to take into account anisotropic nature of image features. Thus, the future work will be twofold. One direction is to apply different diffusion schemes for refining GCF that may enhance

the ability of our approach to deal with the difficulties in OCT. The other one is to extend the current 2-D work to 3-D space that will reconstruct coronary arteries, providing an accurate 3 -D vessel geometry and longitudinal analysis to support the diagnosis of cardiovascular diseases.

\section{References}

[1] D. Huang, E. Swanson, C. Lin, J. Schuman, W. Stinson, W. Chang, M. Hee, T. Flotte, K. Gregory, and C. Puliafito. Optical coherence tomography. Science, 254:1178-1181, 1991.

[2] M. Adhi and J. Duker. Optical coherence tomography - current and future applications. Curr Opin Ophthalmol., 24:213-221, May 2013.

[3] C. Kauffmann, P. Motreff, and L. Sarry. In vivo supervised analysis 
of stent reendothelialization from optical coherence tomography. IEEE TMI, 29:807-818, March 2010.

[4] K. Tung, W. Shi, R. Silva, E. Edwards, and D. Ruechert. Automatical vessel wall detection in intravascular coronary oct. In ISBI, pages 610613, 2011.

[5] S. Tsantis, G. Kagadis, K. Katsanos, D. Karnabatidis, G. Bourantas, and G. Nikiforidis. Automatic vessel lumen segmentation and stent strut detection in intravascular optical coherence tomography. Medical Physics, 39:503-513, January 2012.

[6] S. Celi and S. Berti. In-vivo segmentation and quantification of coronary lesions by optical coherence tomography images for a lesion type definition and stenosis grading. Med. Image Anal., 18:1157-1168, 2014.

[7] T. Adriaenssens et al. Automated detection and quantification of clusters of malapposed and uncovered intracoronary stent struts assessed with optical coherence tomography. J. Cardvasc. Imaging, 30:839-848, 2014.

[8] S. Gurmeric et al. A new 3-d automated computational method to evaluate in-stent neointimal hyperplasia in in-vivo intravascular optical coherence tomography pullbacks. Lect. Notes Comput. Sci., 5762:776-785, 2009.

[9] A. Roy et al. Lumen segmentation in intravascular optical coherence tomography using backscattering tracked and initialized random walks. IEEE J. Biomed. Health Inf., 20:606-614, 2016. 
[10] M. Moraes, D. Cardenas, and S. Furuie. Automatic lumen segmentation in ivoct images using binary morphological reconstruction. Biomed. Eng. Online, 12, 2013.

[11] M. Schaap, L. Neefjes, C. Metz, A. Giessen, A. Weustink, N. Mollet, J. Wentzel, T. Walsum, and W. Niessen. Coronary lumen segmentation using graphcuts and robust kernel regression. In IPMI, pages 528-39, 2009 .

[12] M. Xu, D. Wong, J. Liu, A. Taruya, and A. Tanaka. Graph based lumen segmentation in optical coherence tomography images. In ICICSP, 2015.

[13] J. Cha, M. Farhangi, N. Dunlap, and A. Amini. Segmentation and tracking of lung nodules via graph-cuts incorporating shape prior and motion from 4d ct. J. Medical Physics, 45:297-306, January 2018.

[14] Y. Boykov, O. Veksler, and R. Zabih. Fast approximate energy minimization via graph cuts. PAMI, 23:1222-1239, Nov. 2001.

[15] A. Goldberg and R. Tarjan. A new approach to the maximum-flow problem. Journal of the Association for Computing Machinery, 35:921940, 1988.

[16] Y. Boykov and V. Kolmogorov. An experimental comparison of mincut/max-flow algorithms for energy minimization in vision. PAMI, 26:1124-1137, Sept. 2004.

[17] O. Juan and Y. Boykov. Active graph cuts. In CVPR, pages 1023-1029, 2006. 
[18] G. Slabaugh and G. Unal. Graph cuts segmentation using an elliptical shape prior. In ICIP, pages 1222-1225, 2005.

[19] P. Das, O. Veksler, and Y. Boykov. Semiautomatic segmentation with compact shape prior. $C R V, 62: 28-36$, January 2006.

[20] G. Funka-Lee, Y. Boykov, C. Florin, M. Jolly, R. Moreau-Gobard, R. Ramaraj, and D. Rinck. Automatic herart isolation for ct coronary visulaization using graph-cuts. In ISBI, pages 614-617, 2006.

[21] S. Vicente, V. Kolmogorov, and C. Rother. Graph cut based image segmentation with connectivity priors. In CVPR, 2008.

[22] O. Veksler. Star shape prior for graph-cut image segmentation. In ECCV, pages 454-467, 2008.

[23] Y. Cao, K. Cheng, X. Qin, Q. Yin, J. Li, R. Zhu, and W. Zhao. Automatic lumen segmentation in intravascular optical coherence tomography images using level set. J. Compu. and Mathe. Meth.in Medicine, pages $1-11,2017$.

[24] E. Essa and X. Xie. Automatic segmentation of cross-sectional coronary arterial images. J. CVIU, 165:97-110, 2017.

[25] T. Zhao and D. Ruan. Image segmentation with a novel regularized composite shape prior based on surrogate study. J. Medical Physics, 43:2187-93, May 2016.

[26] H. Zhang, E. Essab, and X. Xie. Graph based segmentation with minimal user interaction. In IEEE ICIP2013, pages 4074-4078, 2013. 
[27] B. Li and S. Acton. Active contour external force using vector field convolution for image segmentation. IEEE TIP, 16:2096-2106, August 2007.

[28] C. Li, C. Xu, C. Gui, and M. Fox. Distance regularized level set evolution and its application to image segmentation. IEEE TIP, 19:3243-3254, December 2010.

[29] K. Zhang, L. Zhang, H. Song, and W. Zhou. Active contours with selective local or global segmentaiton: A new formulation and level set method. Image and Vision Computing, 28:668-676, 2010.

[30] K. Li, X. Wu, D. Chen, and M. Sonka. Optimal surface segmentation in volumetric images-a graph-theoretic approach. PAMI, 28:119-134, January 2006.

[31] H. Zhang and X. Xie. Divergence of gradient convolution: Deformable segmentation with arbitrary initializations. IEEE Trans. Image Processing, 24:3902 - 3914, 112015.

[32] T. Chan and L. Vese. Active contours without edges. T-IP, 10(2):266$277,2001$.

[33] K. He, G. Gkioxari, P. Dollar, and R. Girshick. Mask r-cnn. IEEE TPAMI, 1:1-13, 2018.

[34] X. Xie and M. Mirmehdi. MAC: Magnetostatic active contour. T-PAMI, 30(4):632-646, 2008. 
[35] Hochbaum DS. A new-old algorithm for minimum-cut and maximumflow in closure graphs. J. Networks, 37:171-193, April 2001.

[36] Ketaro Wada. labelme: Image polygonal annotation with python. In https://github.com/wkentaro/labelme, 2016. 